\title{
A case study of foundation failure of a residential building: From diagnosis to reparation
}

\author{
Ahmed Merah \\ University AmmarTelidji of Laghouat, Faculty of Civil Engineering and Architecture, Research Laboratory of Civil \\ Engineering (LRGC), Laghouat, 03000, Algeria. \\ * Corresponding Author: a.merrah@lagh-univ.dz
}

Received: 18-08-2020

Accepted: 13-11-2020

\begin{abstract}
The pathology that appears in reinforced concrete structures, old and new, is inevitable and involves all stakeholders at all levels of responsibility, from the technician to the decision maker at the highest level. It also concerns engineers from design offices and experts from offices responsible for diagnosing existing structures, as well as engineers from repair companies. Like all countries in the world, the study of the pathology and safety of civil engineering buildings is one of the big problems encountered in the field of construction in Algeria. The foundations are among of the structural elements of the buildings most affected by this damage, these constitute the link between the structure and the soil, they allow the transmission of loads from the structure to the soil. If these structural elements are damaged, they affect the general stability of the building. Depending on the severity of the damage, it can cause the building collapse. The main objective of this paper is to study a pathological case of foundation failure of a residential building: from diagnosis to reparation. This repair was carried out according to an intervention plan comprising stages spread over time, this allowed the strengthening of the infrastructure and gave the building an acceptable level of security.
\end{abstract}

Key words: Construction, Pathology case, Diagnosis, Foundation failure, Reinforcement.

\section{Introduction}

The construction of buildings continues to evolve, in materials, techniques and equipment. However, this evolution can generate structural degradations, giving rise to certain pathological cases. the most important of these cases is the pathology of the foundations. this can be defined as disorders in buildings, their causes, consequences and treatment. This pathology is mainly due to two factors: one is the soil, which is the support of the building foundations and must provide a strength equal to or greater than the load imposed by the superstructure; the other represents the entire structure, which must be sized according to the construction rules specific to each country.

Furthermore, the loss of bearing capacity of the foundations of a building is one of the most dangerous pathologies which can cause the total ruin of buildings, this problem concerns all actors in the act of building, such as engineers, designers, realization companies. Moreover, the cost of repairing damaged foundations is very high compared to its initial cost. In his studies Mulyukov (1992) have shown that all damage observed in buildings was linked to foundation pathologies. Furthermore, it was also found that the deterioration of the foundations was due to five main causes, insufficient soil studies, errors in the design of the foundations, the poor quality of the foundations, an unfavorable action on the foundations and a change of geological and hydrogeological site conditions.

In another way, in order to synthesize the main causes of degradation of reinforced concrete structural elements, (Carretero-Ayuso et al., 2016) conducted a statistical study based on the analysis of damages, symptoms and their evolutions, the results of this study showed $66 \%$ of damages are related to foundations and $90 \%$ of these impairments were from walls and isolated footings. 
In the same context, (Thompson and Mechling, 2016), through an experimental companion carried out on the soil of the foundations of two buildings in Colorado, this led to geotechnical recommendations in order to remedy the damage observed on of the two buildings based on hydro compressive soil. The results obtained show that the characteristics of the foundation soil vary over short distances and the high soil moisture can also be the cause of differential settlement.

In addition, (Nelson et al., 2017) studied four pathological construction cases implanted on expansive soil and made the following conclusions:

- Choose the soil samples carefully;

- Perform all regulatory tests on these samples and interpret them properly;

- Achieve adequate drainage,

- Contractors need sufficient knowledge about this type of soil.

As part of post realization, (Vazquez et al., 2016) show through their study that rigor in the design according to the rules of construction and the strict control of the realization of structures in reinforced concrete can avoid many future damages in new constructions.

Concerning also, the causes of buildings degradations, (Nangan et al., 2017) shown that the damages on the foundations is due to various causes, this one being: the poor preparation of the soil, the variation of the soil water content, the presence near the construction of large trees and failures of plumbing systems. These causes generate cracking of walls and partitions.

In order to help the authorities to preserve the performance of buildings, (Dos Santos et al., 2017) analyzed, diagnose, classify and expose the possible causes of the pathologies that have appeared in buildings (public and private) located in Sinop - MT in Brazil and their solutions. The results of this study showed that the cracks were caused first by the construction details, by the action of the temperature, by the poor execution of the works, by the quality of the materials and by the quality of the monitoring of the works. Moreover, the direction and nature of cracks is an important factor to facilitate the diagnosis of pathologies.

The quality of materials used in the phase of structural reparations is more important in their durability and efficiencies, (McDonald et al., 1991).

In the same way, (Mushiri et al., 2017) studied the structural cracks in a hospital in Zimbabwe, through the technical documents, the geotechnical tests, the results of these investigations showed that the cracks were due to the poor quality of the brick masonry, the thick mortar of the masonry and the insufficient depth of the foundations and recommend deep foundations for new construction on this site. Regarding, the repairing operation of damaged structures is a complex step, since it is faced with constraints such as the existing soil, the type of foundation, nearby buildings, loads applied and access to the place of repair. Such constraints lead to the operation becoming costly; hence it is necessary to carry out geotechnical studies to collect the necessary information's on the soil, (Dos Santos et al., 2017).

In another hand, (Guimarães and Delgado, 2015) shows in their study that the excessive humidity of soil is the most cause of buildings degradations

According to (Mulyukov, 1992) for the repair of the pathology of the foundations, there are several types of repair:

Intervention in the foundation soil: when the cause of the foundation degradation is the soil, in this case a soil treatment is carried out in order to increase its bearing capacity. For this type of reinforcement, grout injections are used to densify the soil such as jet grouting and compaction.

Increase in the dimensions of the foundations: according to the following equation, 
$\sigma=F / A$

For a constant force, the stress applied to the ground varies with the surface of the foundation. Consequently, when the pressure applied to the soil reaches a value greater than its bearing capacity, increasing the dimensions of the foundation reduces the stress on the soil.

Micro piles are used to transfer the loads from the structure to another, more resistant layer of soil.

In the case of this study, the last cause is the main factor of degradation of the building namely the change of geotechnical characteristics of the soil, by the permanent presence of water in the soil seat. this water comes either from damaged drinking water or sanitation pipes.

One of the fundamental components of the rehabilitation of structures involves the reinforcement of their foundations. This kind of intervention leads to the increase of structural safety and load capacity.

However, the water is one of the main causes of the damage that can occur on new or old buildings, its presence in initially dry soils causes the soil to lose its mechanical characteristics in this case its bearing capacity. This water usually comes from deteriorated sewer lines, rainwater and sometimes leaky water pipes.

In order to sensitize all stakeholders in the act of building, it is certainly good to design a structure from the structural point of view; nevertheless will it be necessary to provide well designed and maintained sanitation pipes in an efficient manner. As a result, the service life of the structures can be extended without prohibitive additional costs.

Indeed, the cracks that appear in the constructions are warning signs of movement in the loadbearing structure of a structure or these fills that goes from the natural movement remaining in acceptable margins for the exploitation of the structure until alarming movements putting out of use the work even up to its peril.

In reinforced concrete structures, cracks will occur due to the low tensile strength of the concrete. However, the opening of the crack should not be important in order to compromise the durability or appearance of the structural member.

The cracks warn engineers of the existence of a serious problem in the structure, and thus reduce these performances of service and exert psychological effects on their users. It has been noted that structures that are prone to cracking and not reported in time can present hazards to occupants and enormous expense to homeowners.

This study concerns a reinforced concrete building with 3 floors and crawl space situated in Laghouat, Algeria, for residential use, started exploiting in 1985. The structure is designed as a beam column system with foundations in insulated foundations and floors in solid slabs. It is part of a group of six buildings. (Fig. 1).
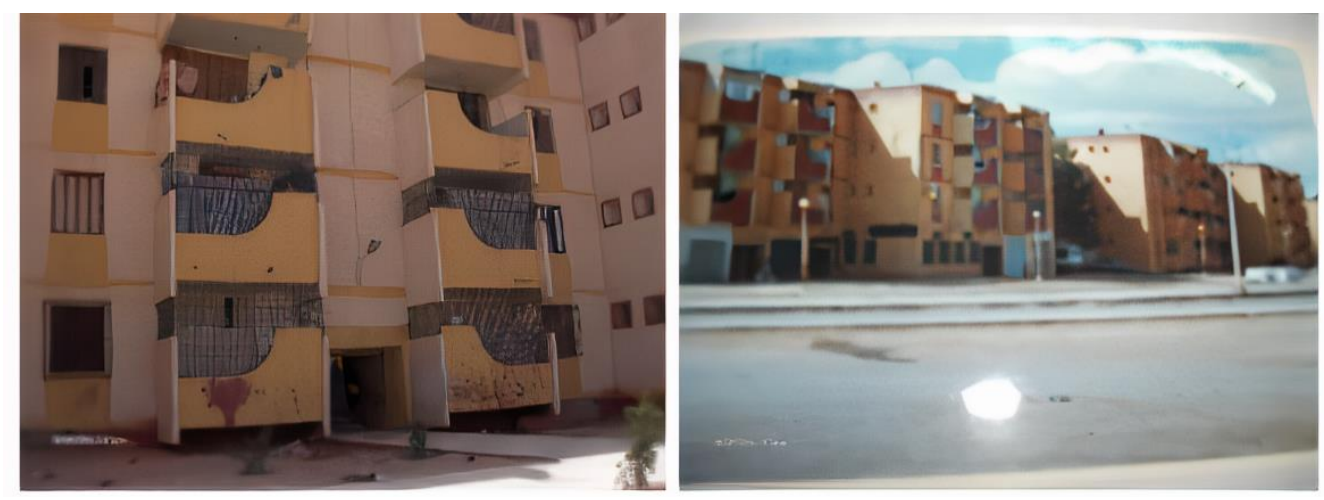

Fig. 1. Building $(R+3)$ 
This study attempts to identify the nature, origin, directions of cracks, intensity and incidence of the failure of foundations, besides diagnose and prognosticate, as well as to verify the structural properties of building and propose the solution of confrontment to lead at a building with an acceptable level of security.

During this study we will present a real case of pathology of foundation failure and through this case we develop the findings until the solutions and their executions. Finally, we present the conclusions obtained as a result of the work performed.

\section{Degradations}

\subsection{Pre-diagnosis}

A pre-diagnosis show the appearance of cracks inclined at $45^{\circ}$ on the exterior walls in double partitions and on the partitions in plaster tiles (Fig. 2), these cracks started appear in 1997.
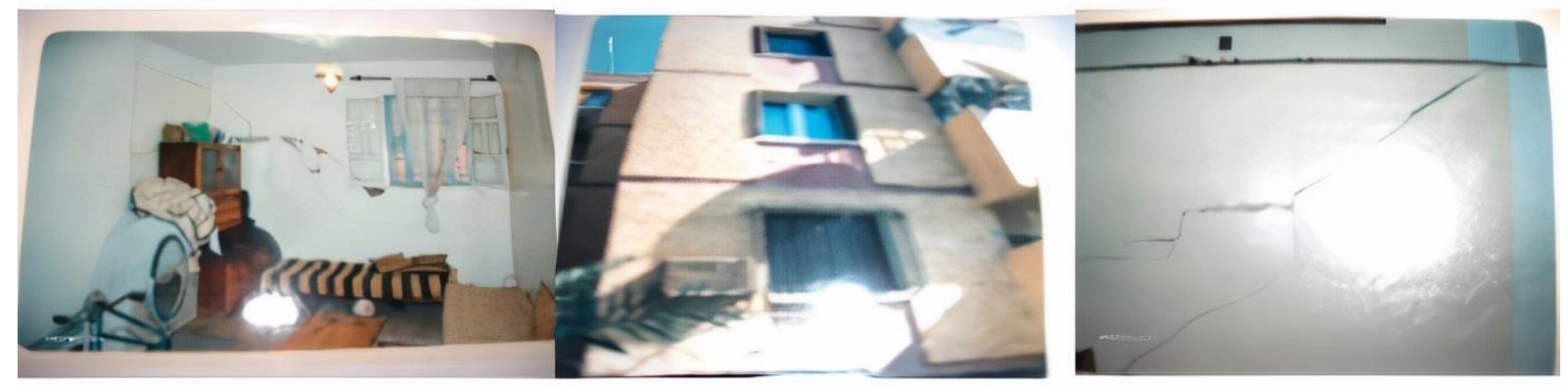

Fig. 2. Cracks in exterior and interior walls

The soil of the crawl space of the building was highly humid, showing a failure in the pipes of drinking water or sanitation.

More ever, permanent stagnation of wastewater inside the crawl space caused by the saturation of pipes as well as the deterioration of the pipes and manholes. Sanitary collectors are heavily degraded in which walls are made walls with masonry blocks.

Some pipes drain into the crawlspace. The nature of the sitting soil is clay nature. From that preceded, the foundations of the building underwent a differential settlement which caused the damage observed, (Burland et al., 1978).

\section{Methodology of diagnosis}

The interventions for diagnostic to be performed must be properly planned before starting reparations. Identifying the factors that can affect the success of the repair is crucial, including: the nature of the soil, identification of the affected structural elements, the soil moisture content, the interior conditions of the building and its surroundings and their respective locations. With the planning and research conducted, the criterion is established to define the correct technique in each case, thus obtaining a greater success in solving the problem. Due to the difficulty and variability of the factors, there is some unpredictability in the results.

The selection of the repair must be supported by a complete diagnosis to know the origin of the pathology, by studying the expected efficiency of the repair and by correlating this with the cost of repair.

The methodology adopted for the diagnosis of the disorders observed is based on in-situ visual examination, and consists in executing the following steps:

- Collect all technical and graphic information of the building (architectural plans, civil engineering plan, date of construction, building materials, type of supporting structure) 
- Take images inside and outside the building

- Inspecting the immediate environment of the building, this inspection makes it possible to detect if there are for example traces of humidity, deteriorated seams of assault, cracks on the buildings of proximity, stagnations of water near the building, rainwater pipes damaged.

- Realization of the witnesses with plaster on the cracks, in order to follow their evolution

- Drill holes in the soil near cracks in walls,

- Perform a geotechnical study to determine the new properties of the foundation soil under the new hydrogeologic conditions.

- Based on the data collected, draw up a plan of action to begin repairing damaged structural elements.

In addition, the pre-diagnosis must make an inventory of the technical and functional situation of the building, as well as its environment. this information facilitates the understanding of pathologies and helps the technician in decision-making to successfully perform the intervention.

\section{Diagnoses}

The diagnosis of construction pathology is mainly based on an in situ visual examination. An improper diagnosis can lead to improper decisions which result in loss of money and time.

In this sense, for a better understanding of the problem lead to correct technical solution. To follow the steps of diagnosis mentioned above, soundings to diagnose foundations were made, after sounding, Fig. 3 shows that the soil was completely saturated with sanitary water. this figure (Fig. 3) clearly shows that the sanitary pipes of the building are damaged, and the soil of foundation is completely saturated.

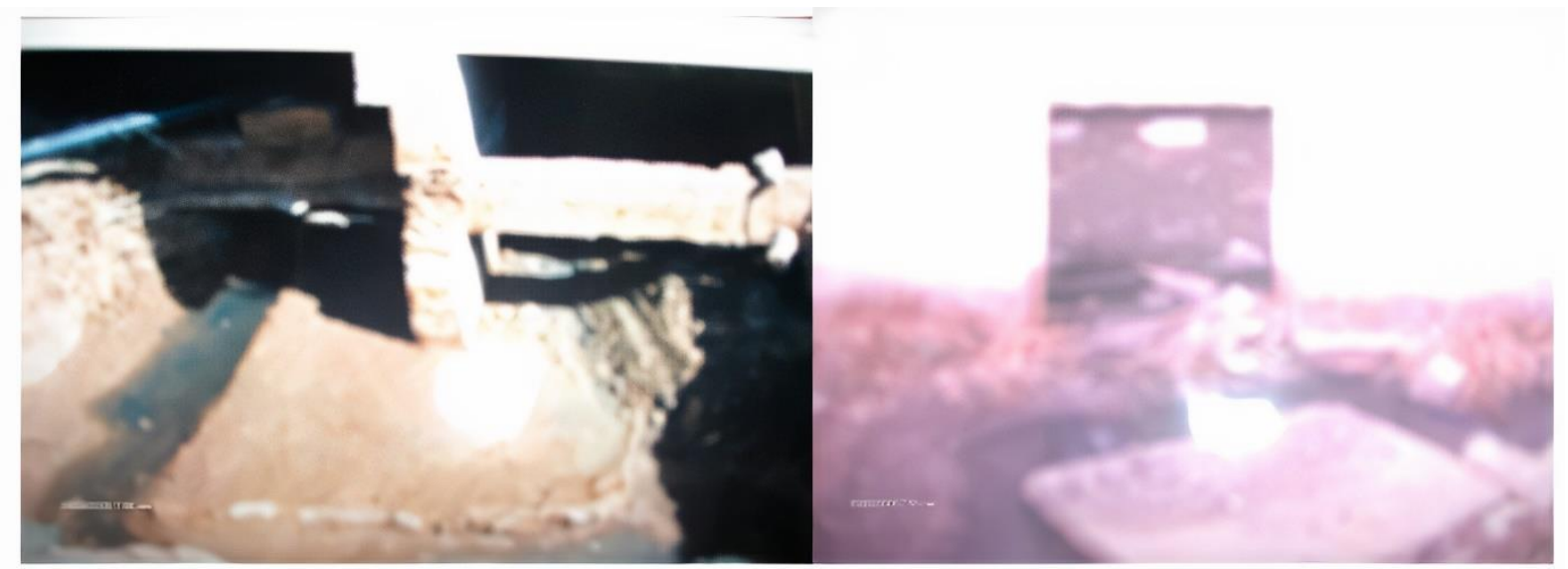

Fig. 3. The soil of foundations is completely saturated and sanitary collector were damaged.

It was recommended that the company remove the spoil under the foundations and repair them alternately to avoid any sudden movement for the building and to ensure the safety of the workers. During the removal of spoil under the first foundations and primers columns, it was found that some of them are heavily damaged (Fig.4). 

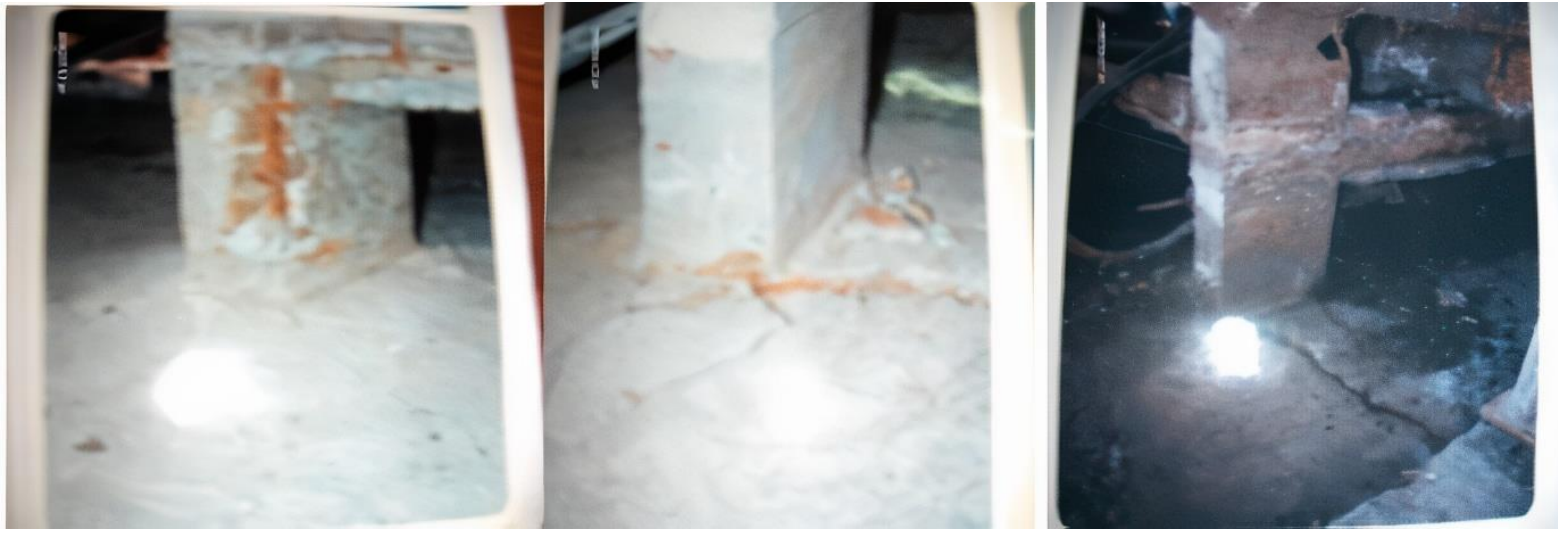

Fig. 4. Primary columns rusted and important cracks in foundation.

In addition, a complementary soil study was recommended. This study shows that the bearing capacity of the soil is reduced with $50 \%$ compared with the initial bearing capacity, caused by the new conditions of change of water content of the ground. Other problems of poor workmanship have been found, namely casting of foundations without formwork or the thickness of foundations have some differences from the plans (Fig.5).
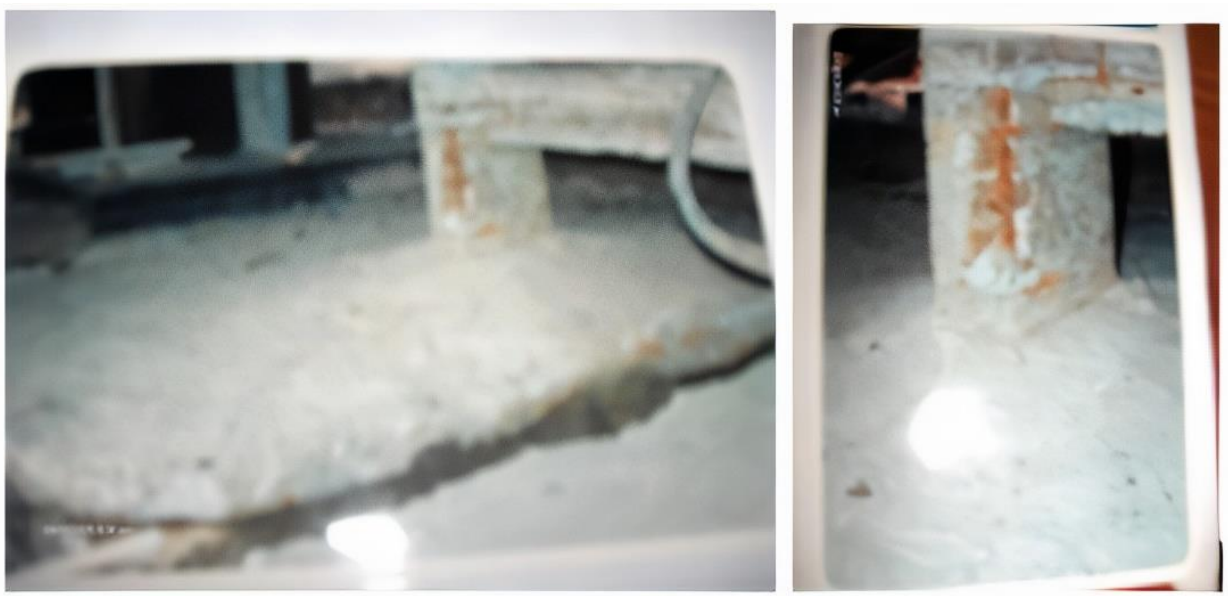

Fig. 5. Foundation casting without formwork.

\section{Reparations}

Among the repair objectives is to re-establish the durability of the concrete, elements and to significantly reduce the rate of deterioration. It should be noted that, in most cases where corrosion of embedded metals is the cause of deterioration, completely preventing future deterioration is not practical.

The main cause of the disorders is the presence of water in the basement of the building which has softened the clay whose characteristics have fallen sharply. The study of the soil in 1980 show that the soil was described as a good soil with a permissible stress of 3 Bars; and advocated the use of a cement type high silica content (HTS). Other elements of poor workmanship have been identified, namely foundations casting in full excavation or the thickness of foundations have some differences from the plans.

Following this it was urgent to activate for the comfort of the infrastructure of the building which threatens ruin at any time.

The first operation consisted of eliminating the main cause of the incident, namely stopping any water supply to the basement of the building. For this, a total deviation of the wastewater evacuation pipes from the building's right-of-way was carried out (Fig. 6) 


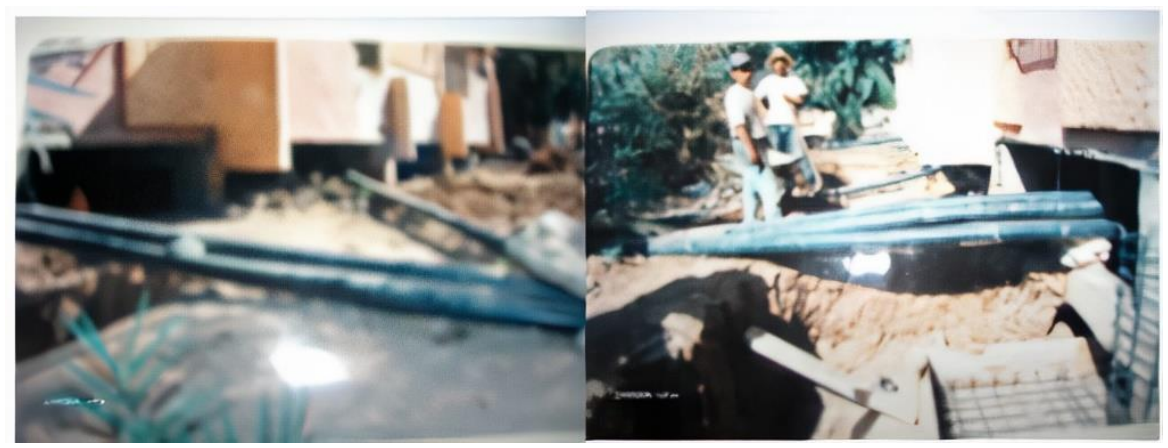

Fig. 6. New sewer lines to evacuate wastewater

The adopted pathology repair methodology is the strengthening of foundations and the primers columns, this repair was carried out by concrete jacketing, (Thompson and Mechling, 2016). The Figs. 7 and 8 show the reinforcement principle.

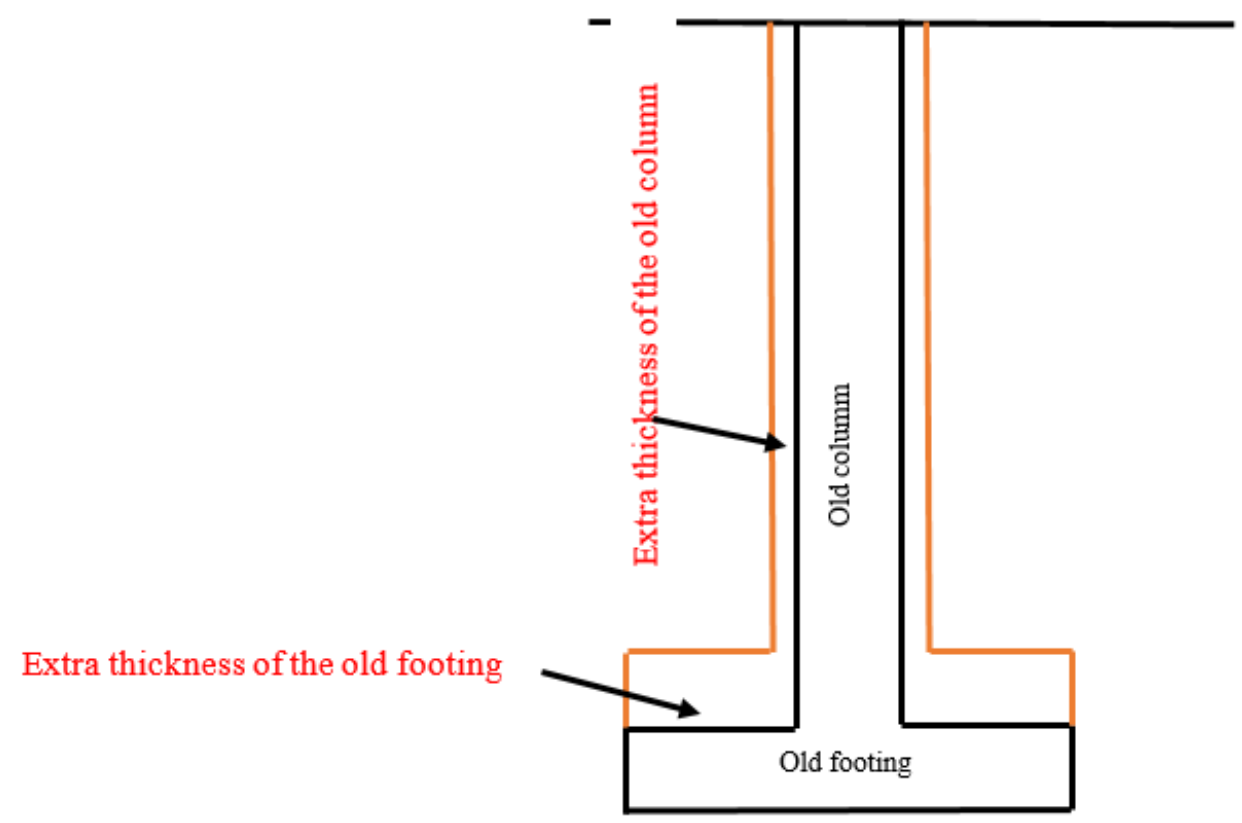

Fig. 7. Foundation reinforcement with thickness increase of the foundation without surface increase

In the first case (Fig.7), the Objectives of these foundation repairs are:

- Reduction of the pressure on the ground under the additional loads as the bending moment (M) and the axial force $(\mathrm{N})$ to the dead weight,

- Increase in bearing capacity (modification of use) reinforcement (degraded) of the foundation,

- Increase rigidity and reinforcement of the foundation.

For cracked foundations and damaged primers columns, take back the loads transmitted, we recommended to carry out foundations and the primers columns with fretting (Fig. 8) 


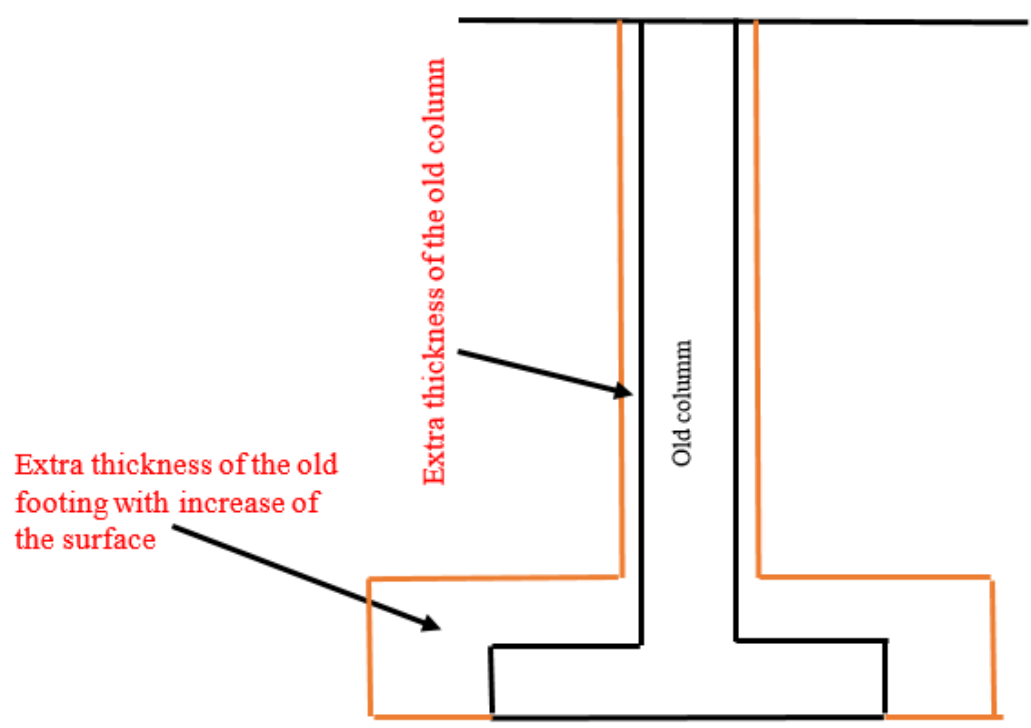

Fig. 8: Reinforcement foundation with increasing the surface and increasing thickness of the foundation

In the second case (Fig. 8), the objectives of this repair:

- increase of section and carrying capacity of the column.

- increase the rigidity of the foundation if the seating area is such that the pressure on the floor is acceptable, (Vlad, 2004) .

In our study the two reinforcement were used, the adopted extra thickness of damaged foundations is $20 \mathrm{~cm}$ with reinforced bars diameter $14 \mathrm{~mm}$ spaced $10 \mathrm{~cm}$, the same thickness is adopted to repair the primers columns, the reinforced transverse frames bars were in $10 \mathrm{~mm}$ spaced with $10 \mathrm{~cm}$ in order to increase their cross-sections.

The new concrete is dosed at $400 \mathrm{~kg} / \mathrm{m}^{3}$ of HTS sulphate-resistant cement with the addition of adjuvant; the contact surface between the old and the new concrete must be brushed to remove any rust (Fig. 9).
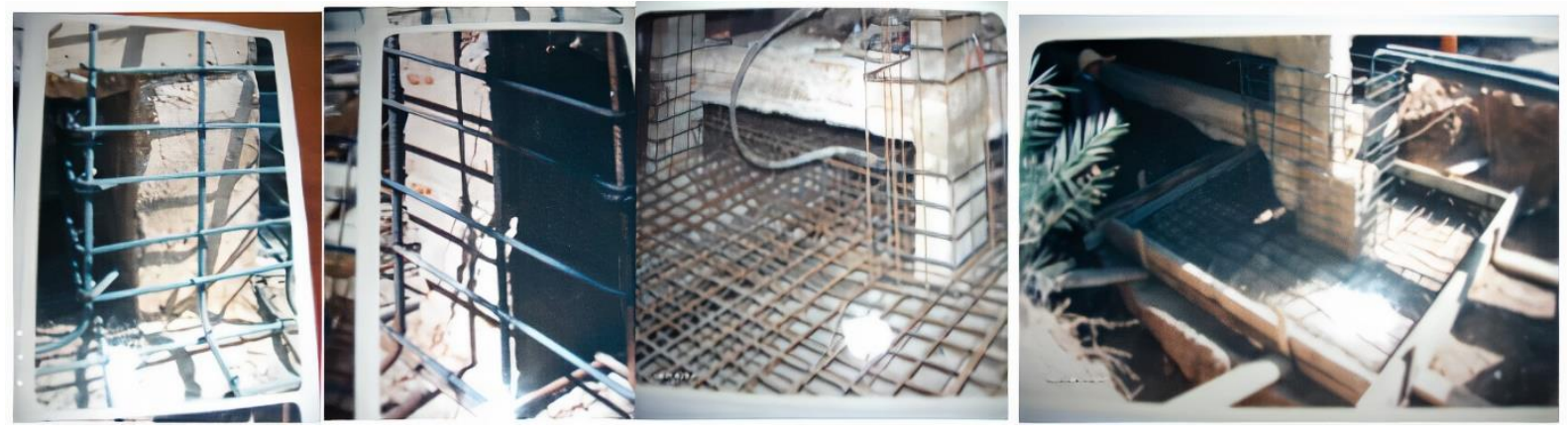

Fig. 9. Reinforcement of the foundations and primers columns

For foundations crushed: To ensure the transmission of loads, we recommended to make a counter foundation with spades and to double the primer columns. In the same way, A wall with concrete was made in the circumferential between the floor and the top floor of the crawl space.

The Fig. 10, shows, the foundation and primer columns after reparation, all foundations are painted with bitumen protection. 


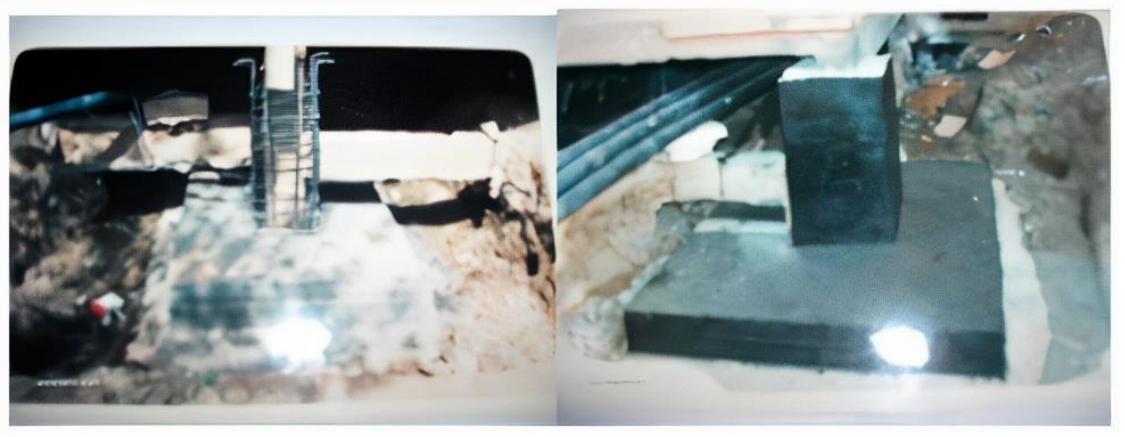

Fig. 10. Foundation and primer columns after reparation

The Fig. 11, shows the building after reparation

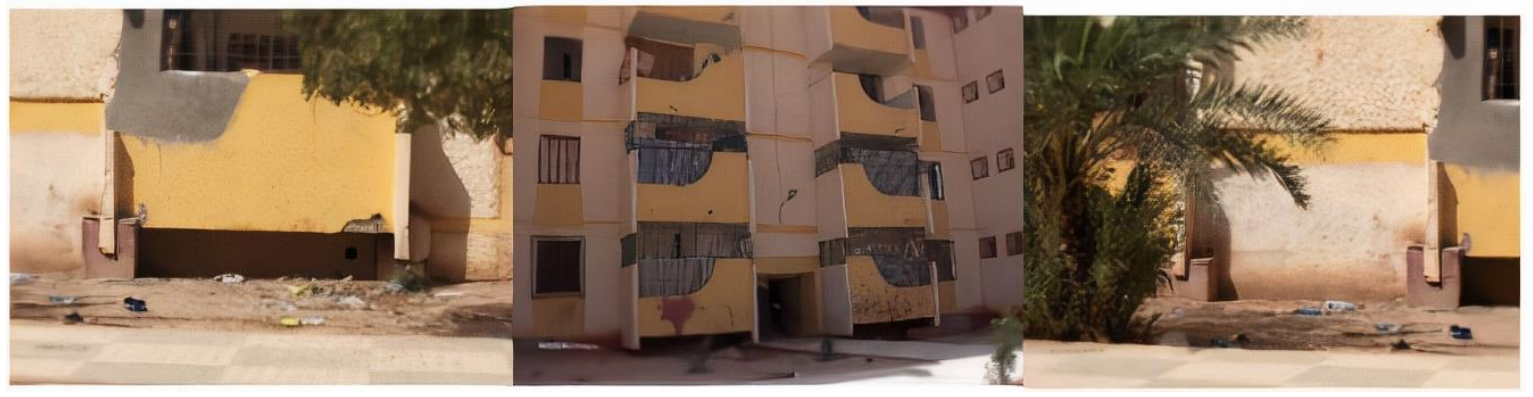

Fig. 11. Building after reparation

\section{Conclusions}

In the conclusion of this study, it was show that the reinforcement of building foundations is a subject with a main importance. This pathology of buildings needs a fast intervention in the site in order to ensure the safety of the structure and its users.

In this way, the success of a foundation's reparation needs the knowledge of the characteristics of the foundation and the ground. Once the geologic and geotechnical study of the soil was performed, in this case, the choosing the best solution to implement becomes easier and the guarantee of success becomes higher. Moreover, the geotechnical study is necessary for all constructions, thereby, all the investment made to study the soil parameters should not be taken as an increased cost, but as an investment.

After four months of these repairs, there was stabilization in the evolution of cracks, as well as the occupants tell us that the cracks are no longer perceptible. The repair of interior partitions and the strengthening of some structural poles have not been started, in order to allow time for the structure to stabilize, and that the soil returns to normal humidity.

In addition, other piezometric measurements will be made to detect the actual level of the water table which must be probably more than 5 meters. In 2000 and after observing that there is no evolution of cracks (by inspection of plasters made after completing the reinforcement of the infrastructure (1998)) the partitions are reconstructed and as well as cracked walls.

In addition, until now the building in question behaves as normally as possible and no visible anomalies were reported.

This is why, we reiterate that not only must special attention be paid to the design and construction of structures, but that a special effort must be made to design and maintain the wastewater and drinking water pipes of the construction, because what has been concluded from the study of this pathological case can be repeated on other blocks if the maintenance of 
the wastewater and drinking water pipes is not done efficiently and if any leak is not repaired in due time.

Besides, a periodical control of the building must be executed after the reparation in order to prevent the pathological processes and failures, and to assure the correct functionality of the building.

\section{References}

Burland, J. B., Broms, B. B., \& De Mello, V. F. (1978). Behaviour of foundations and structures, Geo reports, London, pp. 495-546

Carretero-Ayuso, M. J., Moreno-Cansado, A., \& Cuerda-Correa, E. M. (2016). Research and analysis on recurring anomalies in foundations and structures. Journal of Performance of Constructed Facilities, 30(3), 04015037.

Dos Santos, B. R., Pinheiro, R. V., Arroyo, F. N., de Almeida, D. H., Christoforo, A. L., \& Lahr, F. A. R. (2017). Cracks Studies Case of Buildings in Sinop City-Brazil, International Journal of Materials Engineering, 7(6): 101-110

Guimarães, A. S., \& Delgado, J. M. (2015). Building Pathology and Rehabilitation Techniques-A Rising Damp Treatment Catalogue. Defect and Diffusion Forum, 365, 291-296.

McDonald, J. E., \& McDonald, W. E. (1991). Evaluation and Repair of Concrete Structures : Annotated Bibliography 1978-1988. Volume 2. (Repair, Evaluation, Maintenance and Rehabilitation Research Program). Army engineer waterways experiment station Vicksburg MS structures lab.

Mulyukov, É. I. (1992). Classification of causes of failure of bases and foundations. Soil Mechanics and Foundation Engineering, 29(3), 90-93.

Mushiri, T., Shumba, S., Matora, T., Mhizha, S., Tumbare, M., Taaka, D., \& Musiwa, K. (2017). Investigating Structural Cracks for Infrastructure: Case Study of Anonymous Hospital, in Zimbabwe. EAI International Conference for Research, Innovation and Development for Africa, 432.

Nangan II, A. P., Ganiron Jr, T. U., \& Martinez, D. T. (2017). Concrete Foundation Systems and Footings. World Scientific News, 80, 1-17.

Nelson, E. J., Chao, K. C., Nelson, J. D., \& Overton, D. D. (2017). Lessons learned from foundation and slab failures on expansive soils. Journal of Performance of Constructed Facilities, 31(3), D4016007.

Thompson, R. W., \& Mechling, J. (2016). Geotechnical Engineering for the Remediation of Structures on Collapsing Soils. Geotechnical and Structural Engineering Congress, pp 735-746.

Vazquez, E. G., Haddad, A. N., Qualharini, E. L., Alves, L. A., \& Féo, I. A. (2016). Pathologies in Reinforced Concrete Structures. In Sustainable Construction (p. 213-228). Springer.

Vlad, I. (2004). Case Study on the Foundation and Site Geotechnical Evaluation for the Rehabilitation of an Emergency Hospital Building. International Conference on Case Histories in Geotechnical Engineering. 55. https://scholarsmine.mst.edu/icchge/5icchge/session01/55 